\title{
Inhibitory effect of baicalein combined with gemcitabine in human pancreatic cancer cell lines
}

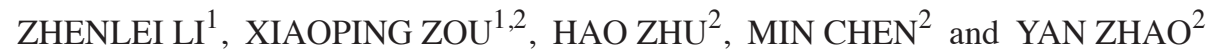 \\ ${ }^{1}$ Department of Gastroenterology, Nanjing Drum Tower Hospital Clinical College of Traditional Chinese and Western \\ Medicine, Nanjing University of Chinese Medicine; ${ }^{2}$ Department of Gastroenterology, Affiliated Drum Tower Hospital of \\ Nanjing University Medical School, Nanjing, Jiangsu 210008, P.R. China
}

Received November 30, 2016; Accepted November 2, 2017

DOI: $10.3892 / \mathrm{ol} .2018 .8043$

\begin{abstract}
Pancreatic cancer is an aggressive disease with a particularly poor prognosis contributing to a substantial percentage of cancer-associated mortality rates. In the present study, the combination treatment of baicalein (BAI) and gemcitabine (GEM) was investigated to examine whether it inhibited the growth of the human CFPAC-1 pancreatic cancer cell line in vitro and in vivo. The cytotoxic interactions between BAI and GEM in human pancreatic cancer cell lines were determined using MTT assays, and the effect of the two agents on apoptosis was detected using Hoechst 33258 staining and annexin V/7-AAD. The protein levels of Bcl-2-associated $\mathrm{X}$ protein (Bax), B-cell lymphoma 2 (Bcl-2), caspase-3, poly ADP ribose polymerase (PARP) and survivin were detected using western blot analysis. Furthermore, the expression levels of Bax, Bcl-2, caspase-3 and survivin in tumor tissues were detected using immunohistochemistry. The results demonstrated that following GEM treatment, the growth of CFPAC-1 cells and xenografts in nude mice were inhibited, and the expression levels of Bcl-2 and survivin were downregulated, whilst the expression levels of Bax, caspase-3 and PARP were upregulated. These effects were enhanced with the use of BAI in combination with GEM. The mechanism underlying the anti-tumor effect of BAI combined with GEM may be associated with the induction of cell apoptosis and the inhibition of proliferation. To the best of our knowledge, this is the first evidence of the efficacy of BAI against pancreatic cancer and may provide the potential clinical evidence for the use of this drug combination for the treatment of patients with pancreatic cancer.
\end{abstract}

Correspondence to: Dr Xiaoping Zou, Department of Gastroenterology, Affiliated Drum Tower Hospital of Nanjing University Medical School, 321 Zhongshan Road, Nanjing, Jiangsu 210008, P.R. China

E-mail: xiaopingzou111@yahoo.com

Key words: baicalein, gemcitabine, pancreatic cancer, apoptosis

\section{Introduction}

Pancreatic cancer is a highly malignant disease, which has the fourth highest mortality rate with a 1-year survival rate of $\sim 29 \%$ globally in 2012, in addition to the fact that the prognosis of this disease is considerably poor (1). Surgery is a potentially curative option for pancreatic cancer, however a majority of patients present symptoms when the tumor has reached an inoperable stage (2). Chemotherapy and/or radiotherapy are the standard treatments for patients with inoperative pancreatic cancer. The standard first line drug gemcitabine (GEM), which is a deoxycytidine analogue, is used to treat patients with advanced pancreatic cancer; however, its efficacy remains low with 5-year survival rates remaining at $20-25 \%$ in $2005(3,4)$. Therefore, there has been considerable interest in combining GEM with other systemic agents in order to improve patient outcomes.

Traditional Chinese herbs may represent a useful resource for drug development (5). Among them, baicalein (BAI) is a purified flavonoid extracted from the root of Scutellaria baicalensis that possesses a wide variety of biological activities, including anti-inflammatory, anti-infection, anti-microbial, neuro-protective and anti-oxidant effects $(6,7)$. BAI has exhibited anti-tumor effects on multiple different types of cancer by inhibiting proliferation and inducing cancer cell apoptosis, including in liver cancer, prostate cancer, lung cancer, breast cancer, bladder cancer and cervical cancer (8-13). The antitumor activity of BAI is mediated by activation of Bcl-2-associated X protein (Bax), B-cell lymphoma 2 (Bcl-2), caspase-3 and poly ADP-ribose polymerase (PARP) (14). Therefore, it is important to evaluate the beneficial effect of BAI as a single treatment or when administered in combination with other conventional anticancer agents in pancreatic cancer.

In the present study, the effects of BAI on pancreatic cancer cells were examined. It was revealed that BAI enhanced the effects of GEM treatment in the inhibition of CFPAC-1 cell viability in vitro by inducing cell apoptosis. In vivo, combination treatment resulted in synergistically reduced tumor volume and weight in a xenograft mouse model. Therefore, the present study identified that combined BAI and GEM treatment may be a promising potential treatment for pancreatic cancer. 


\section{Materials and methods}

Cell culture. Human CFPAC-1 pancreatic ductal adenocarcinoma and PANC-1 pancreatic cancer cell lines were purchased from the Shanghai Cell Bank of the Chinese Academy of Sciences (Shanghai, China). The 2 cell lines were cultured in DMEM (Invitrogen; Thermo Fisher Scientific, Inc., Waltham, MA, USA), supplemented with $10 \%$ fetal bovine serum (Invitrogen; Thermo Fisher Scientific, Inc.), $100 \mathrm{U} / \mathrm{ml}$ penicillin and $100 \mu \mathrm{g} / \mathrm{ml}$ streptomycin in a $37^{\circ} \mathrm{C}$ humidified atmosphere containing $5 \% \mathrm{CO}_{2} / 95 \%$ air.

MTT assay. CFPAC-1 or PANC-1 cells were cultured in 96-well plates at $1 \times 10^{5}$ cells per well. After $24 \mathrm{~h}$, the cells were treated with multiple concentration of BAI alone $(0.1,0.2,0.4$, $0.8,1.6,3.2,6.25,12.5,25,50$ and $100 \mu \mathrm{M})$, GEM alone (0.03, $0.06,0.125,0.25,0.5$ and $1 \mu \mathrm{g} / \mathrm{ml}$ ) or combination treatment $(0.03,0.06,0.125,0.25,0.5$ and $1 \mu \mathrm{g} / \mathrm{ml}$ GEM with either 1.6 or $6.25 \mu \mathrm{g} \mathrm{BAI}$ ) for $48 \mathrm{~h}$, the control group was treated with PBS (pH 7.4). Subsequently, $50 \mu \mathrm{l}$ MTT (1 $\mu \mathrm{g} / \mathrm{ml}$; Sigma-Aldrich; Merck KGaA, Darmstadt, Germany) was added to the cell media and incubated for $4 \mathrm{~h}$ at $37^{\circ} \mathrm{C}$. MTT was discarded and $150 \mu 1 \mathrm{DMSO}$ was loaded in each well. The spectrophotometric absorbance of the samples was measured using a microplate reader (Bio-Rad Model 680; Bio-Rad Laboratories, Inc., Hercules, CA, USA) at $570 \mathrm{~nm}$ with a reference wavelength of $655 \mathrm{~nm}$. The percentage of cell survival was calculated using the following formula: Cell survival=absorbance value of infected cells/absorbance value of uninfected control cells. Six reduplicate wells were measured at each concentration and every experiment was performed at $\geq 3$ times.

Combination index (CI) analysis. CI was used to evaluate the pharmacological interactions of different combinations of BAI and GEM (15). Briefly, synergism, additivity or antagonism in the BAI and GEM combinations was calculated on the basis of the multiple drug effect equation and quantitated by $\mathrm{CI}$, where $\mathrm{CI}=1$ indicates that the two drugs have additive effects, $\mathrm{CI}<1$ indicates synergism effects and $\mathrm{CI}>1$ indicates antagonism effects. The CI was calculated based on: $\mathrm{CI}=(\mathrm{D}) 1 /(\mathrm{Dx}) 1+(\mathrm{D}) 2 /(\mathrm{Dx}) 2+(\mathrm{D}) 1(\mathrm{D}) 2 /=(\mathrm{Dx}) 1(\mathrm{Dx}) 2$, where (Dx) is the dose of the drug, inhibiting ' $\mathrm{x} \%$ '.

Hoechst 33258 staining. Following GEM $(0.125 \mu \mathrm{g} / \mathrm{ml})$, BAI $(1.60$ or $6.25 \mu \mathrm{M})$ or combination treatment, the control group was treated with PBS, the cells were fixed and stained using Hoechst 33258 for $10 \mathrm{~min}$ at room temperature and observed using a fluorescence microscope (Olympus Corporation, Tokyo, Japan) at x20 magnification. A total of 6 fields per treatment were assessed. The stained cells were identified as apoptotic if they were highly condensed with brightly stained nuclei, or non-apoptotic if they were stained pale blue.

Apoptosis assay by flow cytometry. CFPAC-1 or PANC-1 cells treated with $0.125 \mu \mathrm{g} / \mathrm{ml}$ GEM or $1.6 / 6.25 \mu \mathrm{M}$ BAI alone or a combination treatment were incubated for $48 \mathrm{~h}$ at $37^{\circ} \mathrm{C}$, the control group was treated with vehicle solution, and then cells were collected and washed twice with cold PBS and subsequently incubated with Annexin V-FITC/7-ADD double staining solution at room temperature for $15 \mathrm{~min}$.
Then, the stained cells were analyzed using flow cytometry (BD Biosciences, San Jose, CA, USA). The cell apoptotic rates were analyzed using ModFIT software (version 3.2; Verity Software House, Inc., Topsham, ME, USA). Apoptotic events were recorded as a combination of Annexin $\mathrm{V}^{+} / 7-\mathrm{AAD}^{-}$(early apoptotic) and Annexin $\mathrm{V}^{+} / 7-\mathrm{AAD}^{+}$(late apoptotic/dead) events. This experiment was conducted 3 times.

Western blot analysis. Whole cell lysates from CFPAC-1 cells treated with vehicle control or drugs for $48 \mathrm{~h}$, then were washed using PBS once and lysed using RIPA lysis buffer (Beyotime Institute of Biotechnology, Haimen, China), containing protease inhibitor cocktail (Roche Diagnostics, Basel, Switzerland) and phosphatase inhibitor cocktail (Roche Diagnostics), and sonicated for $15 \mathrm{~min}$ on ice. Protein concentration was measured using a BCA protein assay (Bio-Rad Laboratories, Inc.). A total of $30 \mu \mathrm{g}$ samples were separated by SDS-PAGE on a $10 \%$ gel and transferred to polyvinylidene difluoride membranes using the Bio-Rad electrotransfer system (Bio-Rad Laboratories, Inc.). The membrane was blocked using 5\% fat-free milk for $1 \mathrm{~h}$ at room temperature and then incubated with appropriate primary antibody diluted in 3\% BSA solution at $4^{\circ} \mathrm{C}$ overnight. For immunodetection, the following antibodies were used for our analysis: anti-Bax (cat no. 5023), -Bcl-2 (cat no. 15071), -Caspase-3 (cat no. 9662), -PARP (cat no. 9532) and -Survivin (cat no. 2808) antibodies (1:1,000; Cell Signaling Technology, Inc., Danvers, MA, USA), followed by incubation with DyLight dye-conjugated secondary antibodies (cat nos. 35502 and 35568; 1:10,000; Thermo Fisher Scientific, Inc.) for $1 \mathrm{~h}$ at room temperature. Blots were scanned using the Odyssey Western Detection system (LI-COR Biosciences, Lincoln, NE, USA), followed by quantification using ImageStudio software (version 5.2.5; LI-COR Biosciences). Anti- $\beta$-actin (cat no. sc-47778; Santa Cruz Biotechnology, Inc.; 1:1,000 dilution) was used as a loading control.

In vivo model. A total of 24 female BALB/c nude mice (4-5 week-old, 15-16 g) were purchased from the Shanghai Laboratory Animal Center (Shanghai, China). Mice were allowed free access to sterilized water and food and were housed in individual ventilated cages at $23 \pm 5^{\circ} \mathrm{C}$ under a 12-h light/dark cycle. CFPAC-1 cells were resuspended in serum-free DMEM at a concentration of $1 \times 10^{7}$ cells $/ \mathrm{ml}$. A total volume of $0.2 \mathrm{ml}$ cell suspension (total $2 \times 10^{6}$ cells) was then injected subcutaneously into the right anterior armpit of nude mice to establish a xenograft model. The 24 injected mice were randomly divided into 4 groups: control, BAI, GEM and combination treated groups ( $\mathrm{n}=6$ per group). Control group was treated with vehicle solution. The mice were sacrificed at day 28 or when the tumor grew to a maximum of $1,500 \mu \mathrm{M}^{3}$. The Animal Care and Use Committee of Nanjing University of Chinese Medicine ethically approved the experimental protocol.

Immunohistochemical assay. Tumor xenografts were fixed in formalin solution overnight at room temperature and embedded in paraffin blocks, which were sliced into $4-\mu \mathrm{m}$ thick sections for immunohistochemical staining. Following 

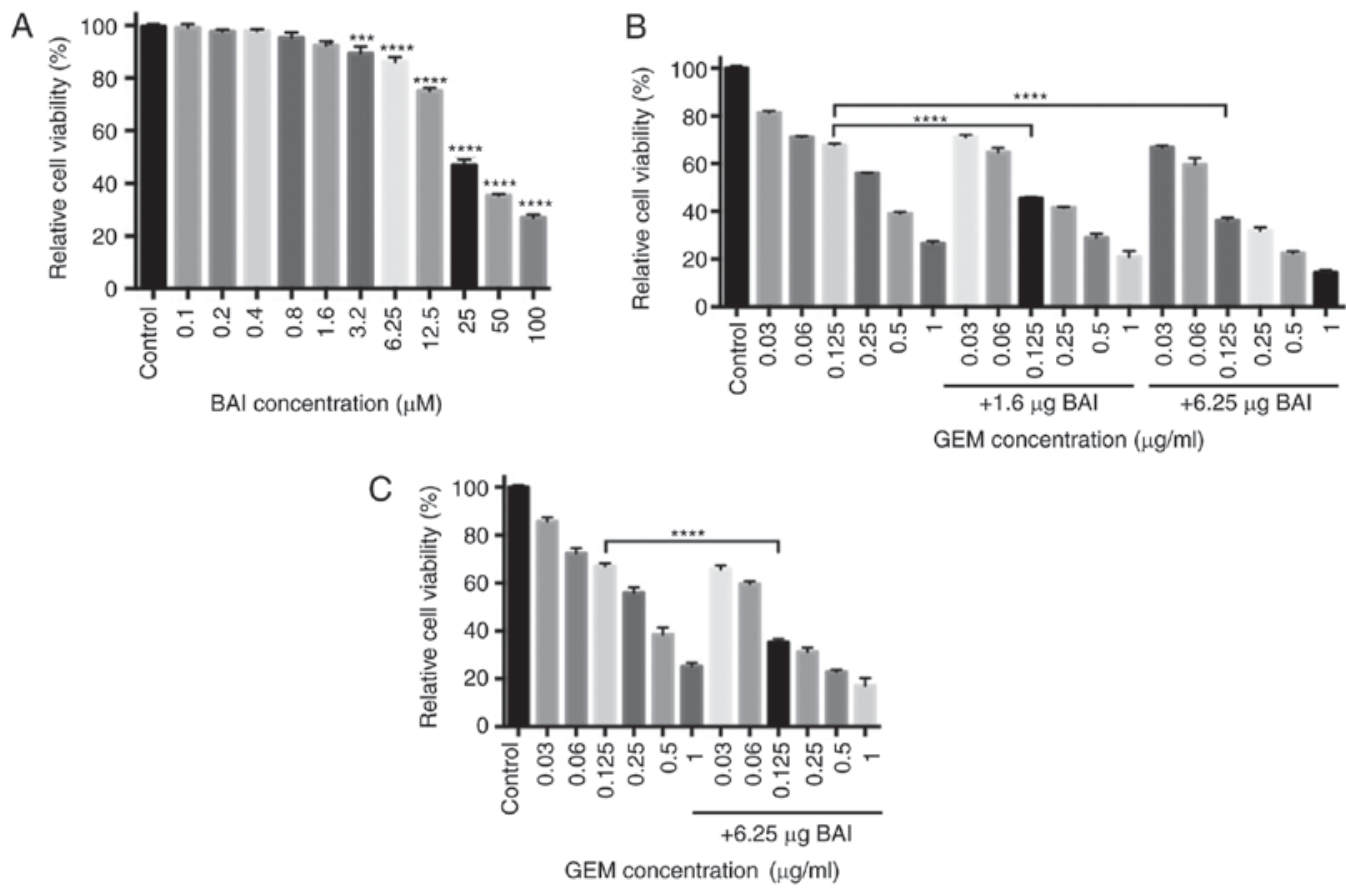

Figure 1. Inhibitory effect of GEM combined with BAI treatment on the cell viability of the human CFPAC-1 and PANC-1 pancreatic cancer cell lines in vitro. (A) MTT assay revealed that an increased BAI concentration (3.2-100 $\mu \mathrm{M})$ significantly decreased cell viability in CFPAC-1 cells compared with that in the control. BAI significantly enhanced the inhibitory effect of GEM treatment in (B) CFPAC-1 and (C) and PANC-1 cell lines. ${ }^{*} \mathrm{P}<0.05$, ${ }^{* * * *} \mathrm{P}<0.001$ and ${ }^{* * * * *} \mathrm{P}<0.0001$ vs. the control or with comparisons indicated by lines. GEM, gemcitabine; BAI, baicalein.

deparaffinization, the sections were incubated at $4^{\circ} \mathrm{C}$ overnight with antibodies of Bcl-2 (cat no. 15071), Bax (cat no. 5023), caspase-3 (cat no. 9662) and survivin (cat no. 2808) (1:500; Cell Signaling Technology, Inc.). The sections were washed 3 times with PBS with $0.1 \%$ Tween-20 (PBST), and incubated for $1 \mathrm{~h}$ at room temperature with a horseradish peroxidase-conjugated goat anti-mouse immunoglobulin $\mathrm{G}$ antibody (1:200; cat no. sc-2031; Santa Cruz Biotechnology, Inc.). The slices were washed with PBST 3 times, and incubated in diaminobenzidine (Sangon Biotech Co., Ltd., Shanghai, China) for $5 \mathrm{~min}$ at room temperature and counterstained with hematoxylin (Sangon Biotech Co., Ltd.) for $1 \mathrm{~min}$ at room temperature. Images were captured using a light microscope (Olympus Corporation) at a magnification of $x 20$. Five sections per tumor tissue were examined.

Statistical analysis. All experiments were repeated at $\geq 3$ times and all data are presented as the mean \pm the standard error of the mean. Graph Pad Prism 6 (GraphPad Software Inc., La Jolla, CA, USA) was use to perform statistical analysis. Comparisons among multiple groups were determined using a one-way or two-way analysis of variance test (two-way analysis of variance was used for tumor volume analysis) followed by Dunnett's or Bonferroni's post hoc test. $\mathrm{P}<0.05$ was considered to indicate a statistically significant difference.

\section{Results}

Synergistic cytotoxicity of the combined treatment of GEM and BAI in pancreatic cancer cell lines. To test the potential of the cytotoxicity of BAI, the human pancreatic cancer cell line CFPAC-1 was incubated with a range concentrations
(0.1-100 $\mu \mathrm{M})$ of BAI for $48 \mathrm{~h}$ and then the number of cells in each group was determined. Treatment with higher concentrations of BAI (3.2-100.0 $\mu \mathrm{M})$ significantly inhibited the viability of CFPAC-1 cells compared with the control group $(\mathrm{P}<0.001$; Fig. 1A). For the cells treated with $0.125 \mu \mathrm{g} / \mathrm{ml}$ GEM, the number of viable cells decreased by $32 \%$ compared with the control group (Fig. 1B). Treatment with BAI in addition to GEM or GEM alone revealed that the viability of CFPAC-1 was inhibited in a concentration-dependent manner. Subsequently, to assess the synergistic effect of GEM in combination with BAI on cell proliferation, CFPAC-1 cells were incubated with a range of concentrations of GEM, in combination with 1.6 or $6.25 \mu \mathrm{g}$ BAI for $48 \mathrm{~h}$. MTT assays revealed that combined treatment of either concentration of BAI with $0.125 \mu \mathrm{g} / \mathrm{ml}$ GEM was significantly more cytotoxic in CFPAC-1 cells compared with the same concentration of GEM alone (CI $(0.125 \mathrm{ug} / \mathrm{ml}$ GEM and $1.6 \mu \mathrm{M}$ BAI $)=0.181$; CI $(0.125 \mathrm{ug} / \mathrm{ml} \mathrm{GEM}$ and $6.25 \mu \mathrm{M}$ BAI $)=0.465$; Fig. 1B). A similar effect was identified in the PANC-1 cell line, where $0.125 \mu \mathrm{g} / \mathrm{ml}$ GEM in combination with $6.25 \mu \mathrm{M}$ BAI significantly decreased the cell viability compared with $0.125 \mu \mathrm{g} / \mathrm{ml}$ GEM treatment alone (Fig. 1C).

BAI and GEM cooperatively induce apoptosis in pancreatic cancer cells. To determine if GEM and BAI treatment induces apoptosis, Hoechst 33258 staining and flow cytometric analysis were used. As indicated in Fig. 2, apoptosis analysis revealed that treatment with GEM or BAI alone induced minimal apoptosis; however, the combined treatment of GEM with 1.6 or $6.25 \mu \mathrm{M}$ BAI resulted in a significant increase of the number of apoptotic cells was observed in CFPAC-1 and PANC-1 cell 
A

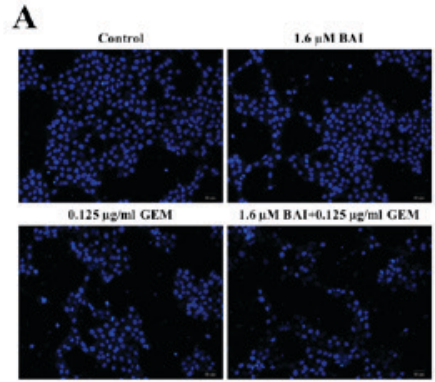

D.

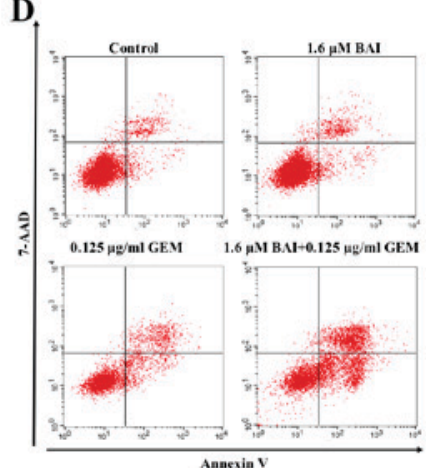

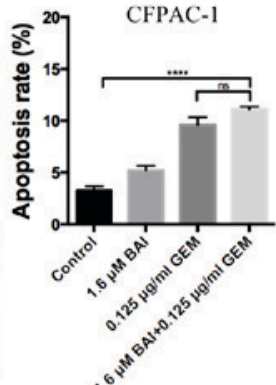

CFPAC-1

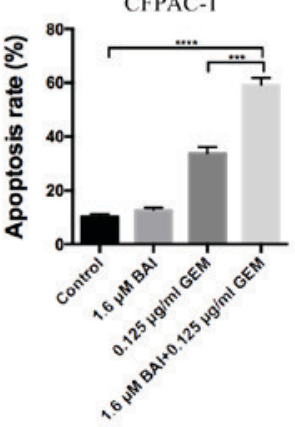

B

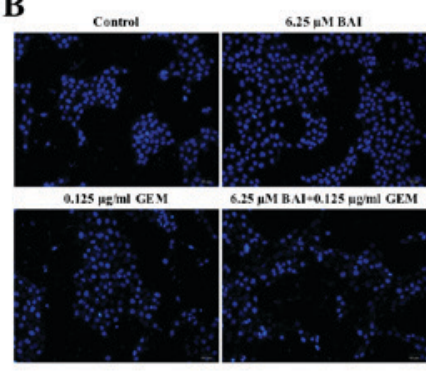

E

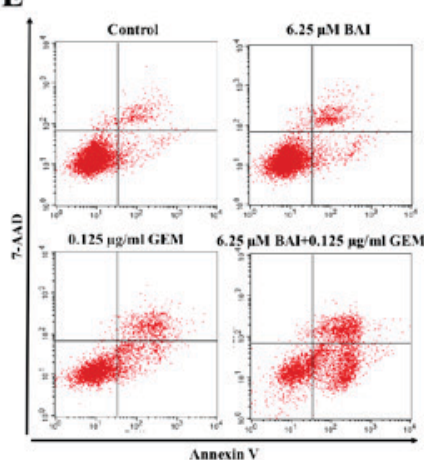

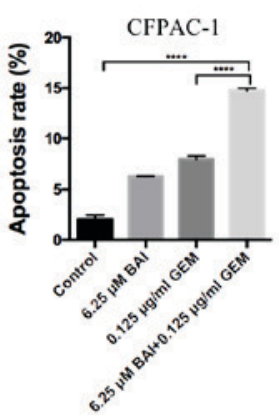
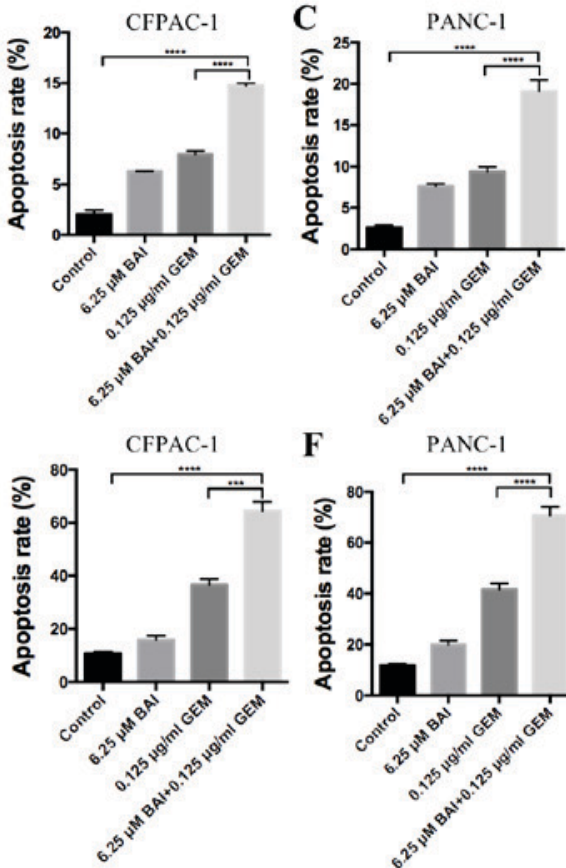

Figure 2. BAI augments apoptosis induced by GEM in human CFPAC-1 and PANC-1 pancreatic cancer cell lines. Morphological analysis of nuclear chromatin was performed using Hoechst 33258 staining in CFPAC-1 cells treated with (A) $1.6 \mu \mathrm{M}$ or (B) $6.25 \mu \mathrm{M} \mathrm{BAI}$, in addition to $0.125 \mu \mathrm{g} / \mathrm{ml} \mathrm{GEM}$ alone or in combination for $48 \mathrm{~h}$. (C) PANC-1 cells were treated with $6.25 \mu \mathrm{M} \mathrm{BAI}$ and $0.125 \mu \mathrm{g} / \mathrm{ml}$ GEM alone or in combination for $48 \mathrm{~h}$ using Hoechst 33258 staining Early and late apoptotic events in the cells were determined using Annexin V/7-AAD staining and flow cytometry staining in CFPAC-1 cells treated with (D) $1.6 \mu \mathrm{M}$ or (E) $6.25 \mu \mathrm{M}$ BAI, in addition to $0.125 \mu \mathrm{g} / \mathrm{ml}$ GEM alone or in combination for $48 \mathrm{~h}$. (F) PANC-1 cells were treated with $6.25 \mu \mathrm{M}$ BAI and $0.125 \mu \mathrm{g} / \mathrm{ml}$ GEM alone or in combination for $48 \mathrm{~h}$ using Annexin V/7-AAD staining. Images were captured using a light microscope at a magnification of $\mathrm{x} 20 .{ }^{* * *} \mathrm{P}<0.001$ and ${ }^{* * * * *} \mathrm{P}<0.0001$ with comparisons indicated by lines. GEM, gemcitabine; BAI, baicalein.

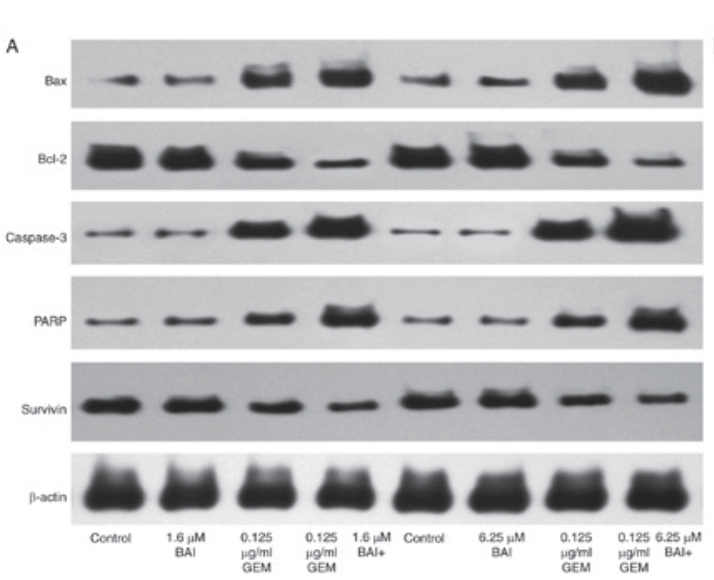

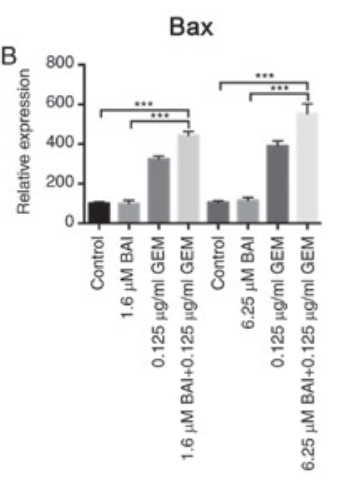

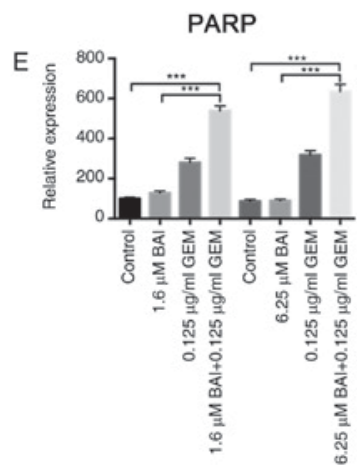

$\mathrm{Bcl}-2$

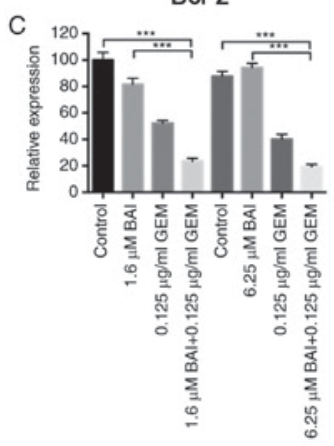

Caspase-3

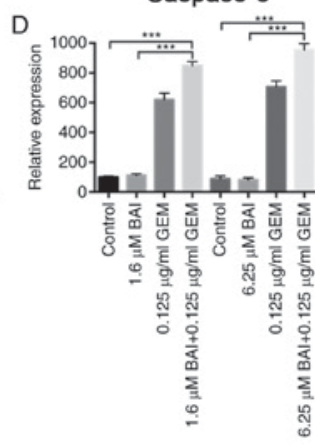

Survivin

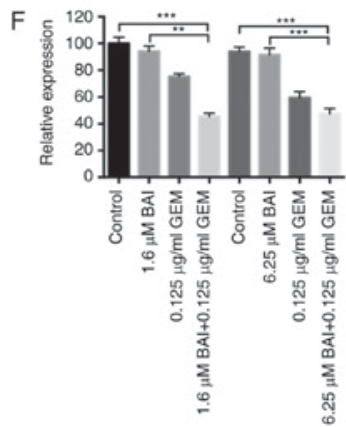

Figure 3. Effects of BAI alone, GEM alone or combined treatment on the protein expression levels of Bax, Bcl-2, caspase-3, PARP and survivin in the human CFPAC-1 pancreatic cancer cell line. (A) Representative images of Bax, Bcl-2, caspase-3, PARP and survivin protein expression, generated using a western blot analysis. $\beta$-actin was used as a loading control. Quantification of the intensity of (B) Bax, (C) Bcl-2, (D) caspase-3, (E) PARP and (F) and survivin. ${ }^{* *} \mathrm{P}<0.01$ and ${ }^{* * *} \mathrm{P}<0.001$ with comparisons indicated by lines. GEM, gemcitabine; BAI, baicalein; Bax, Bcl-2 associated protein X; Bcl-2, B-cell lymphoma 2; PARP, poly ADP ribose polymerase. 


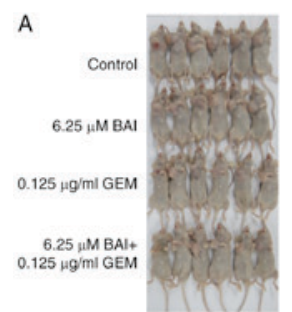

B
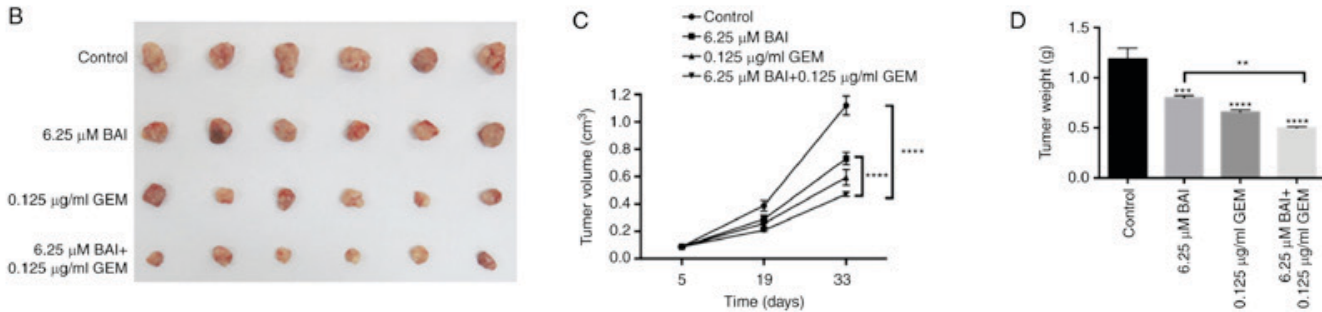

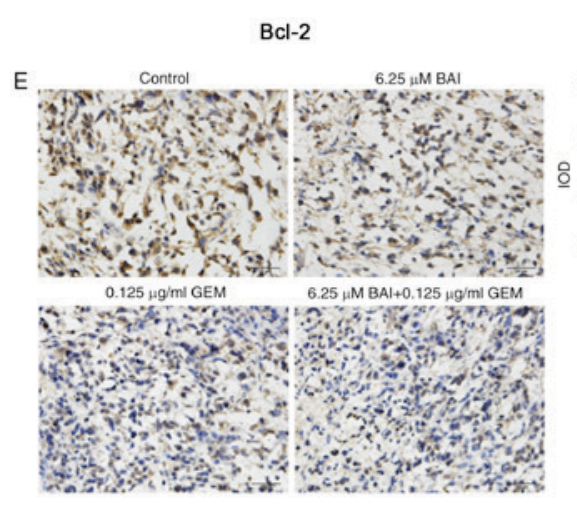

Caspase-3

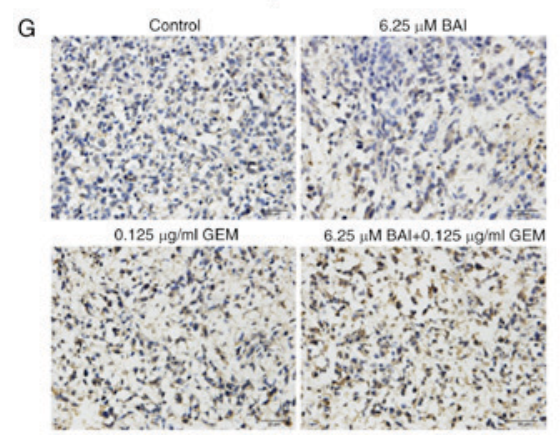

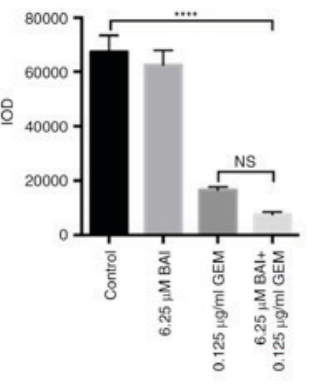
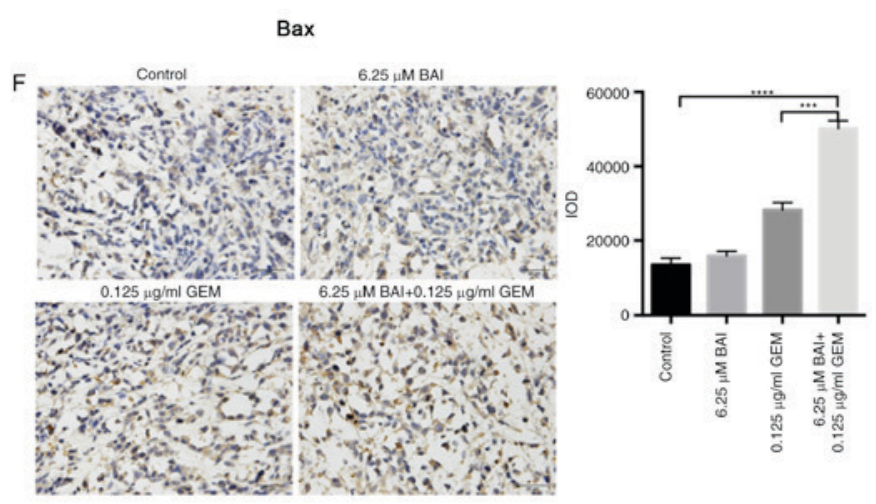

Survivin
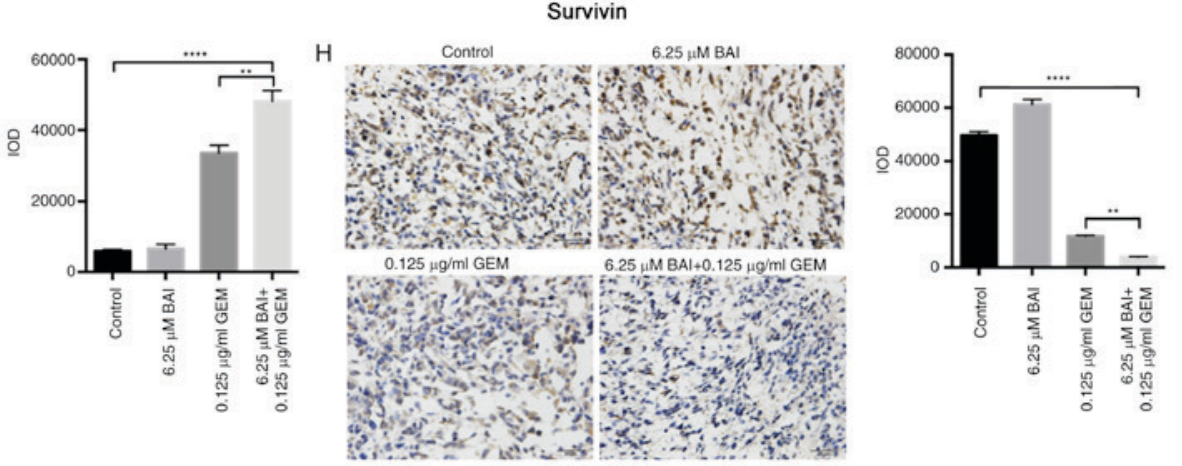

Figure 4. Inhibitory effect of combined treatment with BAI and GEM on the proliferation of the human CFPAC-1 pancreatic cancer cell line in vivo. Representative images of (A) mice and (B) tumors. (C) Tumor volume changes in the xenografts. Resected tumors revealed that combination therapy decreased the tumor volume significantly compared with either the control group or the BAI-alone treated group. The data are presented as the mean \pm SEM. (D) Following 28 days of treatment, the tumor weights were significantly decreased in all 3 treatment groups compared with the control group. The data are presented as the mean \pm SEM. Representative immunohistochemical staining images and quantification for (E) Bcl-2, (F) Bax, (G) caspase-3 and (H) survivin from all groups of mice following treatment with BAI alone, GElM alone or a combination of the 2 treatments. Images were captured using a light microscope at a magnification of $\mathrm{x} 20 .{ }^{* * *} \mathrm{P}<0.01,{ }^{* * * *} \mathrm{P}<0.001$ and ${ }^{* * * * * *} \mathrm{P}<0.0001$ vs. the control group or with comparisons indicated by lines. GEM, gemcitabine; BAI, baicalein; Bax, Bcl-2 associated protein X; Bcl-2, B-cell lymphoma 2; SEM, standard error of the mean.

lines compared with the control $(\mathrm{P}<0.0001$; Fig. 2$)$. In addition, there was a significant increase in apoptosis in cells treated with GEM in combination with $6.25 \mu \mathrm{M}$ BAI compared with GEM alone in CFPAC-1 and PANC-1 cell lines $(\mathrm{P}<0.001$; Fig. 2). Furthermore, western blot analysis demonstrated that the expression levels of Bax, caspase-3 and PARP were significantly increased, and $\mathrm{Bcl}-2$ and survivin were significantly decreased, in the combination BAI (1.6 or $6.25 \mu \mathrm{M})$ and GEM $(0.125 \mathrm{ug} / \mathrm{ml})$ treated group compared with the control group $(\mathrm{P}<0.001$; Fig. 3), and compared with the BAI-alone treated group $(\mathrm{P}<0.01$; Fig. 3$)$. These results provide evidence that the synergistic cytotoxic effects of GEM and BAI against pancreatic cancer cells may be attributed to the induction of apoptosis.

Growth inhibitory effect of BAI and GEM in a mouse model. In the CFPAC-1 xenograft nude mouse model, all mice survived the duration of the experiment with no observable toxic effect. Mice were sacrificed after 28 days and the weights and volume of the xenografts were measured. Compared with the control group, xenografts from the mice treated with BAI or GEM therapy were decreased in volume and weight (Fig. 4A and $\mathrm{B})$. Furthermore, the combination therapy consisting of $6.25 \mu \mathrm{M}$ BAI and $0.125 \mathrm{ug} / \mathrm{ml}$ GEM induced a significant decrease in tumor volume and weight compared with the control and with BAI treatment alone $(\mathrm{P}<0.0001$; Fig. $4 \mathrm{C}$ and $\mathrm{D})$. Immunohistochemical assays additionally revealed that the protein levels of Bcl-2, survivin, Bax and caspase-3 followed a similar pattern to that of the in vitro experiment, since Bcl-2 and survivin were significantly decreased and Bax and caspase-3 were significantly increased in the combined treatment group compared with the control group $(\mathrm{P}<0.0001$; Fig. 4E-H). Furthermore, survivin, Bax and caspase-3 levels were significantly altered in the combination therapy group compared with the GEM-alone treated group $(\mathrm{P}<0.01$; Fig. 4E-H). 


\section{Discussion}

In the present study, the effect of BAI alone and in combination with GEM on the apoptosis and cell viability of the human CFPAC-1 pancreatic ductal adenocarcinoma cell line was investigated in vitro and in vivo. In addition to this, the effects of the combination treatment of BAI and GEM in the PANC-1 cell line in vitro was examined. The mechanism underlying the induction of apoptosis in cancer cells by BAI may be associated with the altered expression levels of pro-apoptotic and anti-apoptotic molecules including Bcl-2, Bax, survivin, PARP and caspase-3. Furthermore, the combination treatment of BAI with GEM significantly reduced the tumor weight and volume relative to either treatment as a monotherapy in the CFPAC-1 xenograft mouse model. In future studies, the growth inhibitory effect of BAI and GEM in a PANC-1 xenograft nude mouse model will be examined. Therefore, the present study investigated for the first time, to the best of our knowledge, the in vitro and in vivo effects of BAI in pancreatic cell lines and in a xenograft model.

BAI, the major bioactive flavone extracted from $S$. baicalensis, is more potent than baicalin, which is hydrolyzed by microflora to BAI (16). So far BAI, at doses that are toxic to malignant cells, have been demonstrated to exert no or little toxicity on normal cells (17). Additionally, BAI has been revealed to inhibit the proliferation of multiple human cancer cell lines $(8,9)$. The anticancer effect of BAI is the induction of apoptotic cell death and the mechanisms include cyclin-dependent kinase modulation, the activation of PARP, and caspase- 3 , caspase-7 and cytochrome c release $(18,19)$. These results indicate that BAI possesses therapeutic potential against cancer.

Apoptosis is an important process for homeostasis by maintaining the balance between cell death and cell survival. The ratio of pro- and anti-apoptotic molecules regulates cell death. Bax is a member of the Bcl-2 protein family associated with apoptosis (20). Bax mediates the permeabilization of the outer mitochondrial membrane and the release of cytochrome c into the cytoplasm, which activates caspase-3 to induce chromosome cleavage and apoptosis (21). Survivin belongs to the inhibitor of apoptosis protein family and is considered a node protein, inhibiting apoptosis and regulating cell mitosis (22). Promising cancer treatment strategies that target apoptotic inhibitors, including Bcl-2 family proteins and inhibitor of apoptosis proteins are presently under investigation (23).

To conclude, in the present study, BAI was revealed to inhibit the proliferation of pancreatic cancer cells due to the induction of apoptosis in vitro and in vivo. Furthermore, evidence was provided that the efficacy of BAI was significantly improved when administered in combination with GEM. Therefore, the present study may provide an alternative strategy for the treatment of pancreatic cancer.

\section{References}

1. Siegel RL, Miller KD and Jemal A: Cancer statistics, 2015. CA Cancer J Clin 65: 5-29, 2015.

2. Stathis A and Moore MJ: Advanced pancreatic carcinoma: Current treatment and future challenges. Nat Rev Clin Oncol 7: 163-172, 2010.

3. Vincent A, Herman J, Schulick R, Hruban RH and Goggins M: Pancreatic cancer. Lancet 378: 607-620, 2011.
4. Boeck S, Ankerst DP and Heinemann V: The role of adjuvant chemotherapy for patients with resected pancreatic cancer: Systematic review of randomized controlled trials and meta-analysis. Oncology 72: 314-321, 2007.

5. Normile D: Asian medicine. The new face of traditional Chinese medicine. Science 299: 188-190, 2003.

6. Qi Z, Yin F, Lu L, Shen L, Qi S, Lan L, Luo L and Yin Z: Baicalein reduces lipopolysaccharide-induced inflammation via suppressing JAK/STATs activation and ROS production. Inflamm Res 62: 845-855, 2013.

7. Kang KA, Zhang R, Piao MJ, Chae S, Kim HS, Park JH, Jung KS and Hyun JW: Baicalein inhibits oxidative stress-induced cellular damage via antioxidant effects. Toxicol Ind Health 28: 412-421, 2012.

8. Chen K, Zhang S, Ji Y, Li J, An P, Ren H, Liang R, Yang J and Li Z: Baicalein inhibits the invasion and metastatic capabilities of hepatocellular carcinoma cells via down-regulation of the ERK pathway. PLoS One 8: e72927, 2013.

9. Guo Z, Hu X, Xing Z, Xing R, Lv R, Cheng X, Su J, Zhou Z, $\mathrm{Xu} Z$, Nilsson S and Liu Z: Baicalein inhibits prostate cancer cell growth and metastasis via the caveolin-1/AKT/mTOR pathway. Mol Cell Biochem 406: 111-119, 2015.

10. Marcinkowski EF, Raz D, Shen B, Xing Q, Yan J, Wen W, et al: Baicalein and meformin decrease small cell lung cancer growth by inhibiting the mTOR pathway in itro. Cancer Res 76: 2183, 2016.

11. Liu Q, Li J, Pu G, Zhang F, Liu H and Zhang Y: Co-delivery of baicalein and doxorubicin by hyaluronic acid decorated nanostructured lipid carriers for breast cancer therapy. Drug Deliv 23: 1364-1368, 2016

12. Choi EO, Park C, Hwang HJ, Hong SH, Kim GY, Cho EJ, Kim WJ and Choi YH: Baicalein induces apoptosis via ROS-dependent activation of caspases in human bladder cancer 5637 cells. Int J Oncol 49: 1009-1018, 2016

13. Peng Y, Guo C, Yang Y, Li F, Zhang Y, Jiang B and Li Q: Baicalein induces apoptosis of human cervical cancer HeLa cells in vitro. Mol Med Rep 11: 2129-2134, 2015.

14. Wang L, Ling Y, Chen Y, Li CL, Feng F, You QD, Lu N and Guo QL: Flavonoid baicalein suppresses adhesion, migration and invasion of MDA-MB-231 human breast cancer cells. Cancer Lett 297: 42-48, 2010.

15. Wang F, Dai W, Wang Y, Shen M, Chen K, Cheng P, Zhang Y, Wang $\mathrm{C}$, Li J, Zheng Y, et al: The synergistic in vitro and in vivo antitumor effect of combination therapy with salinomycin and 5-fluorouracil against hepatocellular carcinoma. PLoS One 9: e97414, 2014.

16. Li-Weber M: New therapeutic aspects of flavones: The anticancer properties of Scutellaria and its main active constituents Wogonin, Baicalein and Baicalin. Cancer Treat Rev 35: 57-68, 2009.

17. Yu C, Zhang Z, Zhang H, Zhen Z, Calway T, Wang Y, Yuan CS and Wang CZ: Pretreatment of baicalin and wogonoside with glycoside hydrolase: A promising approach to enhance anticancer potential. Oncol Rep 30: 2411-2418, 2013.

18. Takahashi H, Chen MC, Pham H, Angst E, King JC, Park J, Brovman EY, Ishiguro H, Harris DM, Reber HA, et al: Baicalein, a component of Scutellaria baicalensis, induces apoptosis by Mcl-1 down-regulation in human pancreatic cancer cells. Biochim Biophys Acta 1813: 1465-1474, 2011.

19. Ling Y, Chen Y, Chen P, Hui H, Song X, Lu Z, Li C, Lu N and Guo Q: Baicalein potently suppresses angiogenesis induced by vascular endothelial growth factor through the $\mathrm{p} 53 / \mathrm{Rb}$ signaling pathway leading to G1/S cell cycle arrest. Exp Biol Med (Maywood) 236: 851-858, 2011

20. Knudson CM and Korsmeyer SJ: Bcl-2 and Bax function independently to regulate cell death. Nat Genet 16: 358-363, 1997

21. Breckenridge DG and Xue D: Regulation of mitochondrial membrane permeabilization by BCL-2 family proteins and caspases. Curr Opin Cell Biol 16: 647-652, 2004.

22. Mobahat M, Narendran A and Riabowol K: Survivin as a preferential target for cancer therapy. Int J Mol Sci 15: 2494-2516, 2014.

23. Hassan M, Watari H, AbuAlmaaty A, Ohba Y and Sakuragi N: Apoptosis and molecular targeting therapy in cancer. Biomed Res Int 2014: 150845, 2014.

This work is licensed under a Creative Commons Attribution-NonCommercial-NoDerivatives 4.0 International (CC BY-NC-ND 4.0) License. 